\section{Folia Phoniatrica \\ et Logopaedica}

Barrett, C. 152

Bernhardt, B.M. 75

Campbell, L. 120

Cleland, J. 120

Crampin, L. 120

Cronin, A. 92

Gibbin, S. 84

Lloyd, S. 120

Mahura, O. 108

Masso, S. 92, 152
McCabe, P. 152

McLeod, S. 73, 92

Mok, Z. 131

Morgan, L. 84

Nelson, T.L. 131

Palo, J.-P. 120

Pascoe, M. 108

Phạm, B. 92

Preston, J. 152

Rossouw, K. 108
Seifert, M. 84

Sell, D. 143

Stemberger, J.P. 75

Sugden, E. 120

Sweeney, T. 143

Ttofari Eecen, K. 131

Verdon, S. 73

Wren, Y. 73, 84

Wrench, A. 120

Zharkova, N. 120

\title{
Subject Index Vol. 72, No. 2, 2020
}

\section{ALSPAC 84}

Assessment 131

Broad transcription 131

Childhood apraxia of speech 152

Children 131

Cleft lip and palate 120

- palate 143

Clinical phonetics 75

Connected speech 152

Crosslinguistic project 75

Cultural and linguistic diversity 92

Curriculum 108

Decolonisation 108
Electropalatography 75

Higher education 108

International phonetic alphabet 131

- Phonetic Alphabet 75

Intervention 143

Multilingualism 108

Narrow transcription 131

Outcomes 143

Paediatric population 131

Percent consonant correct 143

Perceptual analysis 84
Phonemic transcription 131

Phonetic transcription 131

Phonetics 75, 108

Phonology 75

Reliability 84, 143

Speech $84,92,120,143$

- and language therapy 120

- disorders 152

- sound disorder 131

Transcription 84, 92, 120, 131, 152

Ultrasound 75

- tongue imaging 120 